\title{
Successful Corticosteroid-Sparing Effect of Rituximab in the Treatment of Refractory Idiopathic Orbital Inflammatory Disease
}

\author{
Emily H. Shao ${ }^{a} \quad$ Andreas Karydis $^{a} \quad$ Maria Gemenetzi $^{a}$ \\ Simon R.J. Taylor ${ }^{\mathrm{a}, \mathrm{b}}$ \\ ${ }^{a}$ Royal Surrey County Hospital NHS Foundation Trust, Guildford, and ${ }^{\mathrm{b}}$ Faculty of Medicine, \\ Imperial College London, London, UK
}

\section{Key Words}

Rituximab · Idiopathic orbital inflammatory disease

\begin{abstract}
Idiopathic orbital inflammatory disease (IOID) is an idiopathic inflammatory process within the orbit that can result in permanent visual impairment. Although high-dose oral corticosteroids are currently the mainstay of therapy, their long-term usage can cause significant toxicity. We present a case of IOID that was successfully treated with the anti-CD20 monoclonal antibody rituximab following failed steroid sparing with conventional secondline immunosuppressive agents.

(C) 2013 S. Karger AG, Basel
\end{abstract}

\section{Introduction}

Idiopathic orbital inflammatory disease (IOID) is an idiopathic inflammatory process within the orbit that can result in permanent visual impairment. Although high-dose oral corticosteroids are currently the mainstay of therapy, their long-term usage can cause significant toxicity [1]. We present a case of IOID that was successfully treated with the antiCD20 monoclonal antibody rituximab following failed steroid sparing with conventional second-line immunosuppressive agents. 
Shao et al.: Successful Corticosteroid-Sparing Effect of Rituximab in the Treatment of Refractory Idiopathic Orbital Inflammatory Disease

\section{Case Report}

A 61-year-old woman presented with symptoms of left-sided headache, peri-orbital pain and left-sided ocular protrusion. Examination revealed a decrease in her visual acuity from $6 / 6$ to $6 / 24$, which was associated with a left-sided relative afferent pupillary defect. Her left eye was proptotic by $3 \mathrm{~mm}$ on exophthalmometry, with associated ocular pain and conjunctival injection (fig. 1a). Extraocular movements were normal and intraocular examination proved unremarkable.

Blood investigations including thyroid function tests, fasting blood glucose, liver and renal function tests, erythrocyte sedimentation rate and serum ACE were all normal, as was an immune screen including rheumatoid factor, antinuclear antibody and antineutrophil cytoplasm antibody.

A provisional diagnosis of left IOID was made based on the clinical findings and negative blood investigations, and oral prednisolone was started at a dose of $60 \mathrm{mg} / \mathrm{day}$. Her symptoms resolved rapidly and her vision and proptosis improved within days, supporting the diagnosis. However, she suffered a disease relapse when her prednisolone was tapered to $20 \mathrm{mg}$ /day. Subsequent attempts at steroid sparing with mycophenolate mofetil $1 \mathrm{~g}$ b.i.d. and methotrexate $20 \mathrm{mg}$ p.o. weekly proved ineffective over a period of 8 months. The patient started again at $60 \mathrm{mg} /$ day prednisolone together with each second-line agent, but she was unable to get below $20 \mathrm{mg}$ prednisolone without renewed disease activity. Measurement of IgG subclasses at this point demonstrated normal IgG4 levels, although this result may have been confounded by her concurrent treatment with corticosteroids and other immunosuppressive medication.

She then received 2 infusions of rituximab $1 \mathrm{~g}$ i.v. separated by an interval of 2 weeks. This effected disease remission within 4 weeks and enabled her prednisolone to be tapered to $7.5 \mathrm{mg}$ o.d. without relapse over a period of 2 months. Nine months on, she remains in remission on $7.5 \mathrm{mg}$ prednisolone only and maintains a visual acuity of $6 / 6$ with no relative afferent pupillary defect, and no abnormalities on repeat MR scanning (fig. 1b).

\section{Discussion}

Although the pathophysiology of IOID is poorly understood, raised IgG4 levels in serum samples and tissue biopsy of sufferers have implicated IgG4 in its aetiology, although the levels in this case were normal [2,3]. Rituximab (a chimaeric monoclonal antibody against B-cell CD20) has been reported in the successful treatment of refractory orbital disease in granulomatosis with polyangiitis [3, 4] as well as in some cases of IOID [5-7]. Our case differs from previously reported cases in that our patient was sensitive to oral corticosteroids, but it proved impossible to effect steroid sparing with conventional second-line immunosuppressive agents, in contrast to rituximab.

As IOID is rare and presents with heterogeneous clinical manifestations, prospective and randomised evidence investigating the role of rituximab in its treatment is unlikely to occur. Nevertheless, case reports including ours increasingly suggest that rituximab should be considered in refractory IOID unresponsive to conventional immunosuppression or in place of conventional immunosuppression in selected cases. 


\begin{tabular}{l|l}
\hline \multicolumn{2}{l}{ Case Rep Ophthalmol 2013;4:216-218 } \\
\hline DOI: $10.1159 / 000356523$ & $\begin{array}{l}\text { @ 2013 S. Karger AG, Basel } \\
\text { www.karger.com/cop }\end{array}$ \\
\hline
\end{tabular}

Shao et al.: Successful Corticosteroid-Sparing Effect of Rituximab in the Treatment of Refractory Idiopathic Orbital Inflammatory Disease

\section{Financial Support}

S.R.J.T. was supported by the UK National Institute of Health Research. The sponsor or funding organization had no role in the design or conduct of this research and the views and opinions expressed therein are those of the authors and do not necessarily reflect those of the UK Department of Health. The authors report no conflicts of interest. This study was approved by the Royal Surrey County Hospital R\&D Department (12DEV0010).

\section{References}

1 Jacobs D, Galetta S: Diagnosis and management of orbital pseudotumor. Curr Opin Ophthalmol 2002;13:347-351.

2 Lindfield D, Attfield K, McElvanney A: Systemic immunoglobulin G4 (IgG4) disease and idiopathic orbital inflammation; removing 'idiopathic' from the nomenclature? Eye 2012;26:623-629.

-3 Joshi L, Salama AD, Pusey CD, Lightman S, Taylor SR: Rituximab in Wegener's granulomatosis: PR3 titres predict relapse, but repeat treatment can be effective. Ophthalmology 2011;118:2498-2503.

4 Taylor SR, Salama AD, Joshi L, Pusey CD, Lightman S: Rituximab is effective in the treatment of refractory ophthalmic Wegener's granulomatosis. Arth Rheum 2009;60:1540-1547.

5 Schafranski MD: Idiopathic orbital inflammatory disease successfully treated with rituximab. Clin Rheumatol 2009;28:225-226.

6 Kurz PA, Suhler EB, Choi D, Rosenbaum JT: Rituximab for treatment of ocular inflammatory disease: a series of four cases. Br J Ophthalmol 2009;93:546-548.

7 Wallace ZS, Khosroshahi A, Jakobiec FA, Deshpande V, Hatton MP, Ritter J, et al: IgG4-related systemic disease as a cause of 'idiopathic' orbital inflammation including orbital myositis, and trigeminal nerve involvement. Surv Ophthalmol 2012;57:26-33.

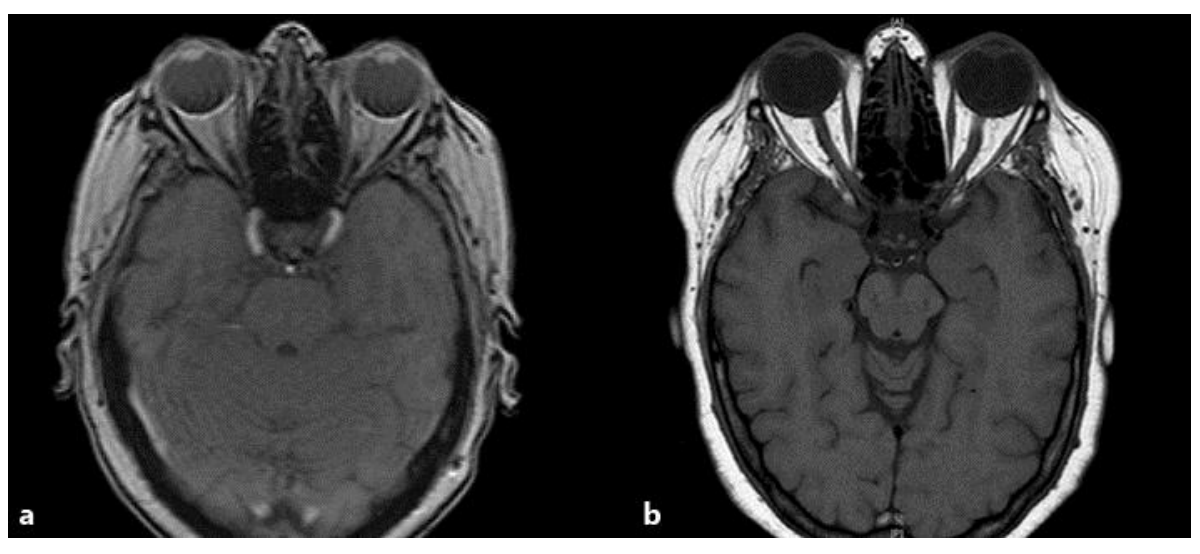

Fig. 1. Transverse MR images of the brain and orbit (T1-weighted images), before (a) and after (b) the treatment with 2 doses of rituximab $1 \mathrm{~g}$ i.v. 